\title{
E1 proceso de etno-racialización y resistencia en la era multicultural: Ser negro en Bogotá ${ }^{1}$
}

\section{Klára Hellebrandová2 \\ Escuela de Altos Estudios en Ciencias Sociales de Paris (EHESS), Francia ${ }^{3}$ \\ klara.hellebrandova@gmail.com}

Recibido: 16 de julio de 2013

Aceptado: 23 de septiembre de 2013

\footnotetext{
Artículo de reflexión basado en una investigación en curso desarrollada dentro de la tesis doctoral en sociologia titulada "Fronteras raciales: ideologia, politicas públicas y experiencia de la racialización en la era multicultural: ser 'negro' en Bogotá", inscrita en la Escuela de Altos Estudios de Ciencias Sociales (EHESS, Paris), dirigida por Eric Fassin (Université Paris VIII), co-dirigida por Mara Viveros Vigoya (Universidad Nacional de Colombia), y parcialmente nutrida de algunas discusiones y sesiones de estudios realizados en el marco del proyecto "Escapando a la desdicha genealógica. El surgimiento y la participación de las clases medias 'negras' en la vida nacional colombiana" dirigido por Mara Viveros Vigoya (Universidad Nacional de Colombia) y financiado por COLCIENCIAS. Código 1101-521-28382. El artículo presenta un análisis crítico de resultados obtenidos a partir de las fuentes primarias (legislación, proyectos), trabajo etnográfico (observaciones, investigación participativa) y entrevistas realizadas a los y las jóvenes afrodescendientes de Bogotá entre febrero de 2011 y mayo de 2013.

2 Magíster en Servicios Públicos y Políticas Sociales de la Universidad de Salamanca, España (2009). Diploma del Instituto de Estudios Políticos de Paris (Sciences-Po I.E.P. Paris) por la mención de Política Comparada, especialidad América Latina (2008) y diploma de Magíster de Investigación del Sciences-Po, I.E.P. París (2008).

3 Estudiante de tercer año del Doctorado en Sociología en la Escuela de Altos Estudios en Ciencias Sociales (EHESS) de París.
} 


\title{
El proceso de etno-racialización y resistencia en la era multicultural: Ser negro en Bogotá
}

\author{
Resumen:
}

El presente artículo trata sobre el proceso de etno-racialización en la era multicultural en el contexto específico de Colombia actual. Se toma como base un amplio marco teórico (racialización, racismo estructural, multiculturalismo, interseccionalidad, racismo cotidiano), el análisis de fuentes primarias y entrevistas en profundidad realizadas con jóvenes afrodescendientes de Bogotá para argumentar que el multiculturalismo, tal como ha sido desarrollado al nivel legislativo y de políticas públicas en Colombia, no reta los fundamentos racializados del poder ni de las relaciones sociales y que existe una variedad de mecanismos que buscan defender el lugar de privilegio de lo blanco-mestizo. Sin embargo y simultáneamente, sugerimos que el multiculturalismo proporciona una serie de posibilidades para cuestionar el sistema social y político racializado y para desarrollar estrategias de resistencia.

Palabras claves: Etno-racialización, Racismo estructural, Racismo cotidiano, Resistencia, Multiculturalismo.

Palabras clave descriptores: Ethnicidad, Negros-identidad racial, Multiculturalismo, Identidad cultural, Discriminación racial, Bogotá (Colombia).

\section{The process of Ethno-Racialization and Resistance in the Multicultural Age: Being Black in Bogota}

\begin{abstract}
This article is about the process of ethno-racialization in the multicultural era specifically in the context of Colombia nowadays. A wide comprehensive theoretical framework is taken as the basis (racialization, structural racism, multiculturalism, intersectionality, everyday racism). The analysis of primary sources and in-depth interviews to young African descents from Bogotá are used to argue that multiculturalism, as it has been developed on a legislative level and in the colombian public policies, is not a challenge for the racialized foundations of power and social relations and that there is a variety of mechanisms that seek to defend the privileges of the white-mestizo. However and simultaneously, we suggest that multiculturalism provides a number of opportunities to question the racialized social and political system and to develop strategies of resistance.

Keywords: Ethno-racialization, Structural Racism, Everyday Racism, Resistance, Multiculturalism. Key words plus: Ethnicity, Black-Racial Identity, Multiculturalism, Cultural Identity, Race Discrimination, Bogotá (Colombia)
\end{abstract}

\section{O processo de etno-racização e resistência na era multicultural: Ser preto em Bogotá}

\begin{abstract}
Resumo
Este artigo trata sobre o processo de etno-racização na era multicultural no contexto específico da atual Colômbia. Baseia-se num quadro abrangente teórico (racização, racismo estrutural, multiculturalismo, intersecionalidade, racismo cotidiano), a análise de fontes primárias e entrevistas em profundidade realizadas com jovens afrodescendentes de Bogotá para argumentar que o multiculturalismo, tal como tem se desenvolvido no nivel legislativo e de políticas públicas na Colômbia, não desafia os fundamentos racializados do poder nem das relações sociais e que existe uma variedade de mecanismos que procuram defender o local de privilégio do branco-mestiço. No entanto, e simultaneamente, sugerimos que o multiculturalismo fornece uma série de possibilidades para questionar o sistema social e político racializado e para desenvolver estratégias de resistência.
\end{abstract}

Palavras-chave: Etno-racialização, Racismo estrutural, Racismo cotidiano, Resistência, Multiculturalismo.

Palavras-chave descritores: Ethnicidad, Identidade Negra-Racial, Multiculturalismo, Identidade Cultural, Discriminação Racial, Bogotá (Colômbia). 


\section{Introducción}

En el presente artículo trataremos el proceso de racialización en la era multicultural en el contexto específico de Colombia actual entendiendo por racialización un proceso de construcción de relaciones de dominación a través de la determinación de diferencias humanas -culturales o fenotípicas- y de las relaciones sociales y por lo tanto, como un proceso dialectico politico, social, cultural y cognitivo de construcción de diferencias que sirven de base para la jerarquización de grupos humanos. Nos concentraremos ante todo en la experiencia del racismo y racialización y en las estrategias individuales y colectivas de resistencia de las personas jóvenes afrodescendientes de Bogotá.

Nuestra propuesta forma parte de una investigación en curso desarrollada dentro del marco de la preparación de la tesis doctoral en Sociologia ${ }^{4}$. Asimismo, la propuesta se nutrió de algunas discusiones y sesiones de estudios realizados en el marco del proyecto "Escapando a la desdicha genealógica. El surgimiento y la participación de las clases medias 'negras' en la vida nacional colombiana"5. Por lo tanto, se trata de una propuesta que incluye resultados parciales de dichas investigaciones.

\section{El multiculturalismo desafiado por la experiencia de las personas afrodescendientes de Bogotá}

La racialización, y la categorización etno-racial que forma parte de ella, son procesos que históricamente estructuran la sociedad colombiana, tanto sus relaciones económicas y de poder, como las relaciones sociales. La ideología del mestizaje y las políticas públicas que la acompañaban, buscaban hacer desaparecer la raza y encarnar la Nación en la categoria mestizo, sin que los fundamentos políticos y sociales racializados de esta fuesen cuestionados.

Sin embargo, la nueva Constitución multicultural de $1991^{6}$, la Ley 70 de 1993 y la legislación multicultural que deriva de ellos, busca

\footnotetext{
4 Tesis doctoral titulada "Fronteras raciales: ideologia, políticas públicas y experiencia de la racialización en la era multicultural: ser 'negro' en Bogotá”. Inscrita en la Escuela de Altos Estudios de Ciencias Sociales (EHESS, París), dirigida por Eric Fassin (Université Paris VIII) y co-dirigida por Mara Viveros Vigoya (Universidad Nacional de Colombia).

5 Dirigido por Mara Viveros Vigoya. Código 1101-521-28382. COLCIENCIAS.

6 Especialmente los artículos 7, 10, 13, 329, 330, y el artículo transitorio 55.
} 
cuestionar este ideal del mestizaje, reconociendo la diversidad cultural y étnica de la Nación y con ella, una serie de derechos específicos de grupos étnicos, especialmente de la población indígena y afrocolombiana. Por lo tanto, el multiculturalismo colombiano propone una nueva forma del gobierno - una forma de biopolitica en los términos de Michel Foucault (1979)- aplicada sin embargo a una sociedad construida sobre fundamentos racializados y racistas que la estructuran en todos los niveles. Así, conviene preguntarnos: ¿Cómo y en qué medida este nuevo paradigma multicultural transforma o cuestiona el proceso mismo de la racialización y por lo tanto, las relaciones sociales y de poder racializadas en específico en el nivel social?

Antes de presentar nuestras hipótesis, es necesario aclarar lo que se entiende por multiculturalismo. Como lo señaló Stuart Hall en su conferencia sobre The Multicultural Question (Hall, 2000), el multiculturalismo es una forma de gobernabilidad -biopolítica en términos de Michel Foucault- que busca administrar sociedades multiculturales -o la multiculturalidad de sus sociedades- con el propósito de reducir las desigualdades y la discriminación estructural de los diferentes grupos. Como lo señala Hall, existen varios tipos de multiculturalismo. Diana Bocarejo y Eduardo Restrepo, en su introducción para el segundo número del volumen 47 de la Revista Colombiana de Antropología (Bocarejo y Restrepo, 2011) definen el multiculturalismo colombiano como etnicista ya que se centra, al nivel legislativo y de políticas públicas, en los grupos étnicos, en particular: los pueblos indígenas y las comunidades negras. Este multiculturalismo etnicista se inscribe y está desafiado por el contexto neoliberal con el modelo de megaproyectos mineros y del conflicto armado que lo reconfigura de manera constante y crucial.

Sin embargo, hay que entender el multiculturalismo como un proceso más amplio, es decir en tanto que las prácticas sociales y políticas lo redefinen y transforman constantemente a través de la agencia de múltiples actores. Es en este sentido que nos interesa analizar la agencia de los jóvenes afrocolombianos de Bogotá que en cierto modo representan lo que Stuart Hall llama "expert vernacular translators" (Hall, 2000, p. 12). Es decir, personas que negocian y transforman los códigos de las fronteras etno-raciales. Así, argumentaremos que el multiculturalismo, tal como ha sido desarrollado en Colombia, no reta los fundamentos racializados del poder ni de las relaciones sociales

\footnotetext{
7 La traducción sería "traductores expertos vernaculares".
} 
y que existe una variedad de mecanismos que buscan defender el lugar de privilegio de lo blanco-mestizo.

No obstante y de manera simultánea, el multiculturalismo provee una serie de posibilidades para cuestionar el sistema social y político racializado y para desarrollar estrategias de resistencia. Analizaremos entonces en qué medida estas aperturas y estrategias contribuyen o no a la de-racialización ${ }^{8}$ y en qué medida esta de-racialización se hace posible a través de la experiencia vivida por las personas jóvenes afrodescendientes de Bogotá. Puede parecer paradójico querer estudiar los efectos del multiculturalismo a través de la experiencia de los afrocolombianos urbanos que no han sido inicialmente incluidos por la Constitución de 1991, ni por la Ley 70. Aún así, argumentamos que es justamente esta fracción de la población afrocolombiana la que desafia, impulsa y transforma el multiculturalismo colombiano al apropiarse de él y al mismo tiempo, la que ha sido afectada por estas transformaciones multiculturales.

Para apoyar nuestra argumentación, dividiremos el presente artículo en dos partes. En la primera buscaremos identificar algunos ejemplos de mecanismos de racialización, del racismo y de la discriminación racial en el contexto actual, preguntándonos sobre la eficacia de los mecanismos legales y de política pública desarrollados en el marco del multiculturalismo. En la segunda parte, queremos visibilizar varios ejemplos de estrategias individuales o colectivas de contestación de este sistema, tanto en la vida cotidiana como en el campo político, activista o artístico. Concluiremos con algunas reflexiones sobre las oportunidades y desafios que presenta el multiculturalismo y la necesidad del reconocimiento del racismo en tanto problema social y político que atañe a todo el país y la sociedad colombiana.

Nuestro marco teórico ha sido bastante amplio y variado por lo que el tema tratado, a saber el proceso de etno-racialización, implica abordar varios niveles de análisis (teórico, político, social, cognitivo, entre otros). Particularmente, en cuanto a la teorización del proceso de la racialización y del racismo estructural, nos basamos en el trabajo de Eduardo Bonilla-Silva (Bonilla-Silva, 1997), Yasmin Gunaratnam

\footnotetext{
8 Por de-racialización entendemos un proceso de de-construcción de las diferencias humanas fenotípicas o culturales- en tanto que base de jerarquización de diferentes grupos humanos. Es decir, se trataria de estrategias que buscan redefinir estas diferencias e invertir la estigmatización etno-racial. En las palabras de Stuart Hall, se trataria de trascender los códigos raciales e invertir el estigma en una identificación positiva: "struggled to insist on and appropriate and transcode their negative figuration into a positive resource.” (Hall, 2000, p. 7). Sin embargo, estas estrategias de inversión conllevan el riesgo de una nueva esencialización.
} 
(Gunaratnam, 2003), David Theo Goldberg (Goldberg, 1993), Howard Winant (Winant, 2000), Glenn Loury (Loury, 2002), y Claire Jean Kim (Kim, 2004), para citar solo algunos.

Todas estas autoras y autores muestran el carácter estructural e histórico del racismo y de la discriminación racial que implican la construcción social de la raza que, a través de sus efectos, representa un principio de visión y división social reflejado en la asignación de significados sociales a los cuerpos humanos. Asi, Glenn Loury, al trabajar sobre el caso estadounidense y en concreto, sobre el tema de la encarcelación de la población afroamericana, busca analizar el proceso histórico de la estigmatización racial y su reproducción y efectos sobre la población racializada como negra hoy en día. A su vez, Eduardo Bonilla-Silva propone una definición integral del racismo que permite entenderlo en tanto proceso histórico estructural que forma un sistema social racializado y penetra todas las esferas de la vida política, económica o social.

En el trabajo de Yasmin Gunaratnam, la autora reflexiona sobre la utilización del concepto de raza proponiendo una metodología para trabajar al mismo tiempo con y contra las categorias raciales y por lo tanto combatir y no corroborar sus efectos. Desde perspectivas y niveles diferentes, estos trabajos muestran que la raza es el resultado de un proceso dinámico que se construye y reconstruye a través el tiempo y el espacio. En este sentido, al analizar la racialización en América Latina y concretamente en Colombia, es importante hacerlo desde la teoría de la colonialidad del poder propuesta por Aníbal Quijano (Quijano, 2000) y desarrollada y ampliada por otras autoras y autores.

Al mismo tiempo, las autoras y autores citados analizan el proceso de cristalización de la raza al nivel social, formando el sentido común racializado. En este sentido es importante entender el multiculturalismo, tal como lo propone Stuart Hall, también como un desafio para nuestro sentido común clásico (Hall, 2000, p. 4), es decir un cuestionamiento de nuestras categorias, teorías y supuestos. Dicho eso, cabe preguntarnos, hasta qué punto el multiculturalismo en Colombia cuestiona y transforma el sentido común racializado.

Al mismo tiempo, al suscribir nuestro trabajo al contexto particular del multiculturalismo en Colombia, nuestra investigación se nutre de diferentes trabajos que no solamente desarrollan el tema del multiculturalismo en Colombia, tal como los trabajos de Claudia Mosquera Rosero-Labbe y colaboradores (Rosero-Labbé, Laó-Montes, y Garavito, 2010), de Carlos Agudelo (2004) o de Peter Wade (1993, 2003, 2010, 
2012). También se revisa el tema de la racialización bajo el contexto del multiculturalismo en los espacios urbanos (Cunin, 2004; Hernández, 2010; Hoffmann, Barbary, y Cunin, 2004; Vigoya y Hernández, 2010) y la racialización experimentada por mujeres afrodescendientes y analizada mediante el concepto de interseccionalidad (intersectionality) (Crenshaw, 1991; Hooks, 1984; Lozano, 2011; Lozano y Sepúlveda, 2005).

El concepto de interseccionalidad se refiere a diferentes maneras de interacción entre la raza y el género, pero también entre otras categorías sociales tales como el sexo, la clase social o la orientación sexual. Así, este concepto nos ayuda a entender la experiencia de las mujeres afrodescendientes que han vivido históricamente en el cruce de diferentes tipos de opresiones y dominaciones. Finalmente, para analizar la experiencia vivida del racismo usaremos el concepto de everyday racism de Philomena Essed (1991) que busca analizar la intersección del racismo y de la experiencia de la discriminación racial al nivel micro y macro a través de las prácticas cotidianas. Así, el concepto de everyday racism "connects structural forces of racism with routine situations in everyday life" (Essed, 1991, p. 2).

Con base en este marco teórico, nuestro trabajo se desarrolla mediante el trabajo etnográfico, el análisis de fuentes primarias (proyectos, documentos oficiales, la prensa) y sobre todo, la realización de entrevistas en profundidad a personas jóvenes ${ }^{9}$ afrodescendientes de Bogotá o viviendo en Bogotá, con acceso a estudios universitarios y pertenecientes a la clase media, todas ellas realizadas entre febrero de 2011 y mayo de 2013.

\section{Cuando la legislación y las políticas públicas se quedan cortas}

Como ya se señaló, el multiculturalismo en Colombia, cuya máxima expresión es la Constitución de 1991 y en el caso de la población afrocolombiana, la Ley 70 de 1993, cuestiona la concepción asimilacionista de la Nación, al otorgar derechos específicos tanto a la población indígena como a la población afrocolombiana y ordenar la aplicación del enfoque

9 Entre 20 y 35 años. Se optó por el trabajo con las personas de esta edad por dos razones principales: la primera es la mayor facilidad para la autora de entrar en contacto con personas de esta edad y por lo tanto conocer mejor su ambiente. Por otro lado, se trata de personas que nacieron o crecieron bajo el multiculturalismo constitucional y por ello, en cierto modo representan una generación puente entre la era del mestizaje y la era multicultural. 
diferencial ${ }^{10}$. Estos derechos se traducen posteriormente en una serie de políticas públicas que buscan reequilibrar la discriminación histórica estructural de estas poblaciones. Sin embargo, el multiculturalismo tal como ha sido planteado por la Constitución de 1991 y la Ley 70, refiriéndose solamente a las "Comunidades negras"11, excluye en gran medida a la población afrocolombiana urbana que muchas veces dificilmente entra en la definición de las "Comunidades negras" establecida por la Ley.

A pesar de la existencia de una legislación bastante desarrollada y una gama de políticas públicas proyectadas (CONPES, 2002; 2010) y a veces implementadas, la situación general de la población afrocolombiana, que sigue mostrando unos de los peores indicadores básicos del país (Cárdenas, Ñopo y Castañeda, 2012; DANE, 2010), no solamente no ha mejorado sino que en varios aspectos -específicamente en los que están vinculados a las consecuencias del conflicto armado, tales como el desplazamiento forzado (Becerra y Ruiz, 2008; Lozano, Hurtado y Silva, 2012)- han empeorado de manera significativa hasta tal punto de alcanzar el estado crítico, varias veces subrayado por la misma Corte Constitucional ${ }^{12}$. La relativa ineficiencia de las politicas públicas y el incumplimiento de las órdenes de la Corte Constitucional están vinculados entre otros factores, a la falta del reconocimiento y del cuestionamiento frente al racismo estructural y la discriminación racial, tanto por parte del gobierno como por parte de la sociedad colombiana en su conjunto.

Lo anterior se puede identificar a través del estudio de caso de la situación de las mujeres afrocolombianas en situación de desplazamiento forzado. Los datos muestran ${ }^{13}$ por un lado, que las mujeres afrocolombianas están en peor situación que las mujeres blanco-mestizas -tanto de manera relativa como absoluta ${ }^{14}$ - y por otro lado, que las mujeres afrocolombianas desplazadas están en peor situación, no solamente frente a las mujeres blanco-mestizas y otras mujeres afrocolombianas

10 Ver en específico AUTO n092 (2008) y AUTO n005 (2009) de la Corte Constitucional relativos a la población afrocolombiana desplazada.

11 Definidas por la Ley 70 (1993), art.2 (5) como sigue: "Es el conjunto de familias de ascendencia afrocolombiana que poseen una cultura propia, comparten una historia y tienen sus propias tradiciones y costumbres dentro de la relación campo-poblado, que revelan y conservan conciencia de identidad que las distinguen de otros grupos étnicos".

12 Ver la Sentencia T-025 de 2004 y los AUTO n 200 (2007), AUTO nº 092 (2008) y AUTO nº 005 (2009) de la Corte Constitucional.

13 Ver: (DANE, 2010) y (Salamanca y Gómez, 2012).

14 Según los datos del DANE (2010) la esperanza de vida al nacer de las mujeres afrocolombianas es de diez años menor que la del resto de las mujeres. 
sino también frente a las mujeres blanco-mestizas desplazadas ${ }^{15}$. Esta situación no se puede entender y por lo tanto corregir de manera adecuada y eficaz sin tomar en cuenta la co-construcción histórica de la raza y del género en el contexto de la colonización y de colonialidad.

Tal como lo muestra María Lugones (Lugones, 2008) basándose por un lado, en la teoría de colonialidad del poder de Aníbal Quijano y por el otro, en el trabajo de la socióloga nigeriana Oyèrónké Oyěwúmí (Oyěwúmí, 1997) sobre la interseccionalidad histórica entre raza, género y sexo, las mujeres negras han sido históricamente despojadas del género y sometidas a una hipersexualización y deshumanización que las puso en una situación diferente a la de las mujeres blancomestizas. Por consecuente, las mujeres afrodescendientes viviendo en esta intersección, sufren de manera diferente y más fuerte, tanto el racismo y la discriminación racial (frente a los hombres afrodescendientes) como el sexismo y el machismo (frente a las mujeres blancomestizas). No obstante, a pesar de esta situación particular que exige acciones específicas, la legislación y las políticas públicas, por un lado siguen homogeneizando a la población afrocolombiana ${ }^{16}$ y por el otro, conciben a las mujeres como un grupo homogéneo ${ }^{17}$, por lo cual en muchas ocasiones las medidas no son, ni pueden ser eficaces para las mujeres afrocolombianas que necesitan una atención con un enfoque diferencial que tome en cuenta la interseccionalidad histórica de género y raza.

Sin embargo, como lo veremos en los siguientes apartados, el multiculturalismo simultáneamente crea y abre ciertos espacios de oportunidad aprovechados por diferentes agentes, en particular los afrocolombianos, para ampliar y desafiar tanto el multiculturalismo como el racismo y el proceso de racialización.

15 Según la III Encuesta Nacional de Verificación de los Derechos de la Población Desplazada, realizada entre el julio y agosto de 2010 por la Comisión de Seguimiento y CID - Universidad Nacional de Colombia, hay en total $49 \%$ de hogares desplazados afrocolombianos con la jefatura femenina, frente a $32 \%$ entre los hogares indigenas desplazados y $41 \%$ entre el resto de los hogares desplazados desde 1998

16 La Ley más importante relativa a la población afrocolombiana, la Ley 70 de 1993, de hecho no menciona a las mujeres de manera específica.

17 Ver por ejemplo la Ley 1257 de 2008 relativa a la violencia contra la mujer que no habla de manera especifica de las mujeres afrocolombianas aunque, por las razones explicitadas anteriormente, estas sufren diferentes formas de violencia vinculadas a su racialización histórica. 


\section{El multiculturalismo en la praxis: ¿Desafiando la racialización?}

Aunque inicialmente la población afrocolombiana urbana como la de Bogotá, no entra en el imaginario multicultural, por la agencia de las mismas personas afrocolombianas, el marco del multiculturalismo se ha ido modificando. De hecho, el multiculturalismo abre o favorece la apertura de un nuevo espacio generando nuevas posibilidades del trabajo para la población afrocolombiana. Estas oportunidades son aprovechadas pero también creadas no solamente por las personas que forman parte de las Comunidades Negras, tal como están definidas por la Constitución y la legislación multicultural, sino también por las personas que no forman parte de estas comunidades.

\section{Aplicando y ampliando el multiculturalismo desde y contra el sistema}

Dada la colonialidad del saber y la prolongada ausencia por un lado, de la enseñanza crítica de la historia colombiana, en específico en cuanto a la colonización, la esclavitud y el racismo, y por el otro lado, la ausencia de los saberes no occidentales en la educación formal, el nuevo paradigma multicultural se enfrenta a problemas de falta de especialistas sobre las problemáticas a tratar. Así, por su propio interés a menudo estimulado por la experiencia vivida de discriminación racial y los efectos del racismo estructural, muchas veces son las mismas personas afrocolombianas las que se convierten en expertos en los temas relacionados con el racismo en sus diferentes formas.

Al mismo tiempo, los afrocolombianos impulsan la integración de esta problemática a las estructuras e instituciones del Estado y en general, a los diferentes campos políticos y de acción social. Es así que en Bogotá por ejemplo, los campos dedicados a la población afrocolombiana y de manera más general al racismo y la discriminación racial son gestionados por las personas afrocolombianas, como en el caso de los proyectos vinculados a la Cátedra de Estudios Afrocolombianos. Sin embargo, muchas veces se trata de puestos de poco poder de decisión y escasos recursos materiales y humanos, lo que habria que interpretar dentro de la falta de seriedad en el replanteamiento de las relaciones de poder inherentes al racismo estructural (Bonilla-Silva, 1997). Es decir, tal como lo apunta Stuart Hall (Hall, 2000), a pesar de la cierta visibilización e inclusión de las personas afrocolombianas en diferentes ámbitos, incluso en los institucionales, las áreas de poder racialmente diferenciadas, siguen casi intactas (Hall, 2000, p. 1). 
Por otro lado, los avances en estos campos -en particular en cuanto a la protección de derechos de la población afrocolombiana- se deben en gran parte al trabajo de las organizaciones afrocolombianas. Así por ejemplo, la Asociación de Afrocolombianos Desplazados (AFRODES) es uno de los actores más importantes en cuanto a la defensa y desarrollo de los derechos de la población afrocolombiana desplazada. De hecho su agencia y logros han sido reconocidos y valorados por la Corte Constitucional que los designa como uno de los interlocutores y referentes a los cuales el gobierno debería consultar a la hora de elaborar políticas públicas para la población afrocolombiana desplazada ${ }^{18}$.

Del mismo modo, AFRODES -como varias otras organizacionescolabora con organizaciones internacionales, tales como (La Agencia de la ONU para los refugiados) o el (Agencia Española de Cooperación Internacional para el Desarrollo) y su trabajo por la defensa de los Derechos Humanos fue premiado en el año 2010 por la Washington Office on Latin America (WOLA) (WOLA's Human Rights Awards Ceremony and Benefit Gala, 2010). De hecho, el trabajo de AFRODES es una muestra importante de la cooperación y simbiosis entre los líderes desplazados de las comunidades afrocolombianas que forman la Junta Directiva de la organización ${ }^{19}$ y el trabajo de los jóvenes afrocolombianas y afrocolombianos con estudios universitarios, una parte de ellos nacida en Bogotá, que brindan a la organización el conocimiento y criterio necesarios para llevar a cabo los diferentes proyectos, informes e iniciativas. Sin embargo, tal como otras relaciones, estas tampoco están desprovistas de tensiones, debates y conflictos inherentes a las dinámicas de un grupo con heterogeneidades generacionales, de género, clase social, origen geográfico, etc.

Así las cosas, bajo una lógica propiciada por el mismo multiculturalismo tal como fue diseñado por la Constitución de 1991 y la Ley 70 , en varias ocasiones mis entrevistados -en este caso hombres afrocolombianos de Bogotá con estudios universitarios que trabajan en las organizaciones afrocolombianas- afirmaron haberse sentido ocasionalmente subestimados por no estar considerados -en su ámbito de trabajo- como los auténticos afrocolombianos. Se entra entonces en un juego de "ser

\footnotetext{
18 AUTO de Corte Constitucional nº 005 (2009).

19 Para saber más sobre los miembros de la junta ver: http://www.afrodes.org/index. php?option=com_content\&view=article\&id=127:staff\&catid=91:quienes-somos\&Itemid=847
} 
Negro $^{20}$ " $y$ ser "visto como negro ${ }^{21}$ ". En este sentido, uno de mis entrevistados -de 26 años, nacido en Bogotá, con estudios universitarios, en un proceso de ascenso social y auto-identificado como Negro-afrocolombiano- muestra esta diferencia comparándose con un compañero de trabajo proveniente de la costa pacífica.

Al hablar sobre una reunión relativa a la población desplazada con los representantes del Estado y los representantes indígenas, mi entrevistado hace en el primer momento una distinción entre la población indígena y la población afrocolombiana con la cual se identifica (nosotros). Al hacer esta distinción aludiendo a que la población afrocolombiana ha sido desatendida por el gobierno y las organizaciones internacionales frente a la población indigena, mi entrevistado marca al mismo tiempo una diferencia entre él y la población afrocolombiana de la costa. Dice entonces que aunque estas reuniones están signadas por muchos estereotipos raciales sobre la población negra, él, gracias a su acento y su manera de comportarse, puede ser percibido como cualquier rolo y por lo tanto no es visto como negro. Al mismo tiempo, añade que eso no le podría pasar a su compañero que viene de la costa pacífica ${ }^{22}$.

Con lo anterior, nuestro entrevistado insinúa que es posible invertir o al menos neutralizar los estereotipos y prejuicios raciales eliminando los estereotipos étnicos: así, poco importarian los estereotipos y prejuicios (falta de organización, falta de educación, falta de seriedad, etc.) que tienen las personas presentes en la reunión sobre la gente negra. Nuestro entrevistado -a pesar de su color de piel- gracias a su educación, su cultura y otros atributos -podría demostrar lo contrario y por lo tanto neutralizarlos. Al contrario, para su compañero no sería el caso aunque viviese en Bogotá desde hace años. Argumentamos, que este caso es un ejemplo del riesgo que conlleva el multiculturalismo, es decir, el riesgo de esencialización etno-racial a partir del lugar de origen. Junto con Peter Wade, quien afirma que el "multiculturalismo [funciona] como mecanismo para reconocer a las "culturas" de una forma limitada, sin realzar el problema del racismo que yace debajo" (Wade, 2012, p. 22), proponemos que el multiculturalismo, en vez de desafiarlo, corre el riesgo de reconfigurar el sentido común racializado que se sigue utilizando y reproduciendo -en muchos casos inconscientemente- incluso por quienes combaten el racismo.

\footnotetext{
20 Al utilizar la $\mathrm{N}$ mayúscula nos referimos a una categoría de auto-identificación utilizada por nuestros entrevistados.

21 Al utilizar la cursiva indicamos que se trata de una categoría racial socialmente construida y dinámica que cambia con el contexto histórico.

22 Extraído de las notas del diario del campo (20 de marzo 2013),
} 


\section{Las estrategias de contestación del sistema social racializado}

Por otro lado, con su presencia activa en el escenario político, social o artístico, pero también en la vida cotidiana, las y los diferentes agentes urbanos desafian y amplían la definición de lo afrocolombiano y muestran hasta qué punto no solamente hacen parte de la población afrocolombiana y de su contribución a la construcción de la sociedad nacional, sino que también son objeto de discriminación racial y del racismo tanto estructural como social, aunque este último se manifiesta de formas diferentes que el que padecen las Comunidades Negras, afrocolombianas, palenqueras y raizales, tal como están definidas por la Constitución.

Para ilustrar estas estrategias individuales o colectivas de la contestación del orden socio-racial, hablaremos de algunos ejemplos por un lado, del campo artístico y por el otro, del racismo cotidiano al nivel social vivido y desafiado por nuestros entrevistados y entrevistadas.

\section{Denunciando a través $y$ con el arte}

$\mathrm{El}$ arte puede representar un medio poderoso, profundo y sensible de lucha contra el racismo a través de su denuncia y visibilización. Para ilustrar la contestación del sistema social racializado a través del arte, proponemos hablar de dos proyectos concretos: el primero creado por la artista blanco-mestiza, Margarita Ariza Aguilar y el segundo por un joven artista afrocolombiano, Fabio Melecio Palacio Prado. A través de su trabajo artístico, ambos muestran el sutil funcionamiento de la racialización y del racismo así como sus efectos sobre la vida cotidiana de las personas racializadas.

\section{Proyecto Blanco Porcelana de Margarita Ariza Aguilar}

La ganadora de la Beca de creación y circulación en artes plásticas de la Secretaría de Cultura de Barranquilla, 2011, la artista barranquillera Margarita Ariza Aguilar, que actualmente vive en Bogotá, presentó su proyecto Blanco Porcelana por primera vez en el sistema de transporte de Barranquilla, Transmetro en 2011. Este proyecto buscaba desvelar el racismo cotidiano en la esfera íntima de la familia y las prácticas de belleza sesgadas por el ideal del blanqueamiento en tanto prácticas de opresión que hacen parte de las relaciones sociales y de poder racializadas, pero que son invisibilizadas y naturalizadas a través de una serie 
de prácticas, expresiones o imágenes que hacen parte de nuestra vida cotidiana. Para lograr este objetivo, la artista buscaba:

$-(\ldots)$ trasladar escenas o fragmentos de mi historia personal fuera de su contexto natural e intervenir objetos del ámbito familiar, situando lo íntimo en un espacio transitable. Trabajo con elementos de mi cotidianidad, sin aparente importancia y mediante cruces con la historia, la apropiación de imágenes y la intervención de espacios, establezco conexiones con aspectos problemáticos de la realidad social. (Aguilar, 2011) $)^{23}$

Así, Margarita Ariza muestra las prácticas cotidianas del blanqueamiento que reproducen el racismo y las relaciones raciales en todos los niveles, afectando en diferentes grados y con diferentes efectos, a toda la sociedad colombiana.

La artista analiza el proceso de blanqueamiento como parte del proceso de racialización y del racismo desde su carácter invisible, que penetra los ámbitos más íntimos de la vida cotidiana, familiar y social. El proyecto muestra la formación del sentido común racializado desarrollado bajo la ideología del mestizaje, que impone el ideal blanco -que en sí es una categoría racial-como el único modelo al cual hay que aspirar. Así, mostrado desde esta perspectiva, el blanqueamiento se impone más que escoge, a las personas desde su primera infancia y con él se instituye también un sentido común racializado que jerarquiza a las personas según su asignación a un determinado grupo racial. La capacidad de la artista de visibilizar el racismo, a través del análisis del proceso del blanqueamiento, como un fenómeno profundamente arraigado que afecta a toda la sociedad en su conjunto y que se reproduce desde lo más cotidiano e intimo, nos parece un aporte importante para el cuestionamiento y contestación al racismo.

Al mismo tiempo, sin que la artista lo expresase, el proyecto Blanco porcelana muestra el funcionamiento y los efectos del racismo desde la interseccionalidad. El proyecto, cuyas diferentes acciones giran alrededor del ideal de belleza blanca visibiliza el efecto desproporcionado del blanqueamiento sobre las mujeres. Dicho de otra manera, como ya lo habiamos visto en el apartado anterior, la categoría mujer y con ella la categoría belleza, en el contexto de colonización occidental y colonialidad de poder, se desarrollan a manera de categorías racializadas como

${ }^{23}$ Este texto es citado del trabajo no publicado de la autora, cuya citación fue autorizada por ella. 
blancas. Este impresionante proyecto visibiliza y critica la aspiración a la blancura heredada de la colonia dentro de las familias colombianas de manera sutil y profunda. Asimismo la obra de arte y la percepción de la sociedad que supone también son una muestra de mecanismos que favorecen y fortalecen la perpetuación del racismo en la sociedad colombiana: el silencio y la percepción del racismo como un fenómeno individual y voluntario.

En efecto, después de haber presentado el proyecto de Blanco Porcelana en Bogotá, Margarita Ariza recibió quejas de algunos de sus familiares que consideran que la artista "puso como símbolo de racismo a nuestra querida madre" (entrevista con Margarita Ariza Aguilar, 14 de noviembre 2012), figura central del proyecto, la abuela materna de Margarita cuya piel habria sido descrita en la familia como "blanco porcelana". Estas quejas y las respuestas de Margarita, que intentaba explicar que no se trataba de usar a la abuela como un símbolo del racismo sino al contrario denunciar las prácticas del racismo estructural a través de su propia vivencia, terminaron en una acción de tutela interpuesta por varios de sus familiares ${ }^{24}$ por violación de derecho a la intimidad y calumnia. El juzgado falló a favor del accionante y el proyecto Blanco Porcelana fue parcialmente censurado. Concretamente, fueron censuradas todas las imágenes y referencias a la abuela.

Este caso y la decisión del juzgado son muy dicientes de varios retos y desafios que tiene que enfrentar el multiculturalismo. Se trata en realidad de un caso transversal que pone en relieve varias problemáticas. De hecho, el juzgado se encontraba frente a un dilema importante en el contexto multicultural, a saber, defender el derecho individual de los familiares de Margarita Ariza frente a la lucha contra el racismo y por lo tanto, la defensa de los derechos colectivos de toda la población afrocolombiana y la que puede estar afectada por él. Así, al fallar en contra del proyecto que busca visibilizar y denunciar el racismo en tanto ataque a los derechos individuales y colectivos fundamentales, el juzgado dio prevalencia a los derechos individuales frente a los colectivos.

Al mismo tiempo la decisión del juzgado subraya un riesgo importante: el de convertir las denuncias y acusaciones del racismo en un ataque contra el buen nombre de la persona y por lo tanto, caer en la victimización de las personas que cometen el racismo o la discriminación racial, que puede ser directa o indirecta, pero también voluntaria o involuntaria. De la forma en que el juzgado 36 penal

\footnotetext{
${ }^{24}$ Acción de tutela interpuesta por Rafael Aguilar Quijano y otros (Acción Tutela No. 20120047).
} 
municipal de Bogotá abordó la tutela se sigue invisibilizando el problema del racismo en tanto problema estructural y lo convirtie en un asunto meramente individual y voluntario.

Fabio Melecio Palacio Prado: De izquierda a derecha (Pradera, 2002)

Fabio Melecio Palacio Prado es un joven artista afrocolombiano que a través de su obra cuestiona las construcciones cognitivas racializadas de las y los colombianos. Al mismo tiempo, el artista muestra la riqueza y profundidad de la cultura afrodescendiente y su importancia y protagonismo en la construcción de la sociedad colombiana, tal como lo hizo por ejemplo, en su "Proyecto los BMR (Bamba, Martillo y Refilón)", nominado al VI Premio Luis Caballero y presentado en 2011 en la Galería Santa Fe del Instituto Distrital de las Artes en Bogotá.

Fabio Palacio cuestiona y hace cuestionar a los espectadores sus propias construcciones, prejuicios y estereotipos sobre la población afrodescendiente, reflexionando sobre el poder de la palabra y las miradas sesgadas por los estereotipos vistos como instrumentos poderosos de la exclusión social. En uno de estos proyectos titulado De izquierda a derecha que consiste en un registro fotográfico tomado en Casa Color de Palmira entre Junio y Julio del 2002, el autor documenta su propia transformación que lo llevó a experimentar el racismo y la discriminación racial de manera más explícita y presente:

-Durante nueve meses me dejé crecer el cabello y recibí todos los sinónimos más desagradables, ese juzgamiento hizo sentir dentro de mi tanta incertidumbre y me llevó a pensar en la frialdad que existe en nosotros los seres humanos, esa manera de señalar, clasificar y estereotipar la imagen del otro. Ahora estoy sentenciado por el poder de la palabra, esa realidad que nos rodea y arrincona la identidad a la expresión más vil: “desechable”. (Prado, 2012, p. 13)

En este proyecto que se basa en su propia experiencia cotidiana, el autor analiza un componente del racismo cotidiano poco estudiado, dificilmente abordable y sin embargo crucial: la mirada. De Izquierda a Derecha exterioriza y analiza uno de los mecanismos cotidianos de racialización y racismo, no siempre voluntario y algunas veces no-consciente. Se trata de un mecanismo que se construye en la intersubjetividad dentro del contexto racializado y racista. El poder racializante de la mirada que provoca sentimientos de inferioridad no se puede explicar sin tomar en cuenta el funcionamiento estructural 
del racismo, analizado por Frantz Fanon en su libro Piel Negra, Máscaras Blancas (Fanon, 1973). Así, tal como lo afirma el autor:

[...] es el hecho de vivir en una sociedad que posibilita su complejo de inferioridad, en una sociedad que basa su consistencia sobre el mantenimiento de este complejo, en una sociedad que proclama la superioridad de una raza: es exactamente en la medida en la que esta sociedad le pone las dificultades [al sujeto racializado como negro], que lo pone en una situación neurótica. (Fanon, 1973, p. 108) ${ }^{25}$

Estos sentimientos pueden de hecho expresarse en una estrategia que busca minimizar el proceso de racialización, por ejemplo a través de la hipernomatividad, es decir, una auto-vigilancia permanente de su propio comportamiento, el estilo de vestirse, la forma de peinarse, etc., que buscaria cumplir al máximo las normas sociales. Sin embargo, el hecho de vestirse de manera elegante por ejemplo, no siempre busca minimizar o esquivar el racismo, pues, tal como lo expresaron varios de mis entrevistados -en este caso hombres afrocolombianos jóvenes de Bogotá- aparte de la búsqueda de una cierta estética, el hecho de vestirse de manera elegante los puede poner en una situación de cierta ventaja: "muchas veces se les ve [a las personas] que quisieran discriminarme pero como no saben muy bien quién soy, porque no saben dónde ubicarme, no lo pueden hacer" ${ }^{26}$. Esta situación no impide y posiblemente tampoco minimiza el racismo -como lo sugiere Fanon "una sociedad es racista o no lo es" y por lo tanto tampoco puede haber un racismo más suave o más fuerte, pero sí puede adoptar diferentes formas de expresión- sin embargo, sí facilitaría su desafio, que puede incluir o expresarse en el desafio de la mirada.

Esta violencia, explícita o implícita, directa o indirecta, es parte inherente del racismo y es experimentada pero también desafiada en diferentes formas y niveles a través de la experiencia vivida del racismo cotidiano. Como lo muestra Philomena Essed a través de su concepto racismo cotidiano (everyday racism), el racismo estructural se traduce en todos los niveles y se reproduce en prácticas y acciones cotidianas, muchas veces invisibles o invisibilizadas. Sin embargo, tal como lo argumenta la autora, la vivencia

25 La traducción es mia: "c'est qu’il vit dans une société qui rend possible son complexe d'infériorité, dans une société qui tire sa consistance du maintien de ce complexe, dans une société qui affirme la supériorité d'une race; c'est dans l'exacte mesure où cette société lui fait des difficultés, qu'il se trouve placé dans une situation névrotique" (Fanon, 1973, p. 108).

26 Extraído de una charla informal con un amigo afrocolombiano de Bogotá, de 33 años, clase media (Notas de diario de campo, el 15 de abril 2013). 
de este racismo cotidiano genera un conocimiento específico del racismo que permite a las personas afectadas reconocer el racismo que muchas veces es solapado o velado.

Para las personas racializadas como negras esta experiencia implica estar sometidas a situaciones difíciles de discriminación, humillación y racismo de forma cotidiana y causa una frustración frente a las pocas posibilidades de protegerse de ellas. Por consecuente, el racismo genera un cierto sentido de desconfianza y violencia latente pero permanente, tal como lo expresa una de mis entrevistadas:

-Y entonces, yo vivo muy frustrada, porque yo siento que yo vivo con mucha rabia pero que en el momento que me hacen las cosas no reacciono con la violencia que me gustaria. Para sacar la violencia de mí. Y me tengo que restringir. Y aparte de eso se me cobra. Entonces, yo siento, que de alguna manera me he acostumbrado, como con los piropos en la calle, como con otro tipo de agresiones. (mujer afrodescendiente de Bogotá, entrevistada el 23 de diciembre 2011 en Bogotá)

En este sentido, es importante subrayar una vez más la experiencia vivida de la organización diferenciada de género en términos raciales (Lugones, 2008) que implica para las mujeres negras una hypersexualización y una violencia cotidiana en diferentes formas (verbal, fisica, simbólica etc.). Varias de mis entrevistadas mencionaron el sentimiento de vulnerabilidad de sus cuerpos, en específico de su cabello y la cola, que son las partes del cuerpo más violentadas en los espacios públicos, como la calle por ejemplo ${ }^{27}$.

La sensación y presencia de la violencia generada es tan invasiva que influye sobre las decisiones a la hora de escoger la vestimenta, de manera que se opta no solamente por no llevar la falda deseada, sino también llevar un abrigo más largo por ejemplo. Sin embargo, esta vulnerabilidad y violencia se siente también cuando se trata del cabello. Tal como lo devela Margarita Ariza en su proyecto Blanco Porcelana, las prácticas del blanqueamiento físico a través del aliser del pelo o el uso de las cremas son muy invasivas y agresivas, no solo fisicamente sino también para la propia identidad y autoestima de las personas y en específico, de las mujeres afrodescendientes que tienen que luchar por el reconocimiento de su feminidad.

27 Taller interno del Colectivo de Feministas Afrodiaspóricas (Notas del diario de campo, 13 de abril 2013). 
Sin embargo, sugerimos que en la época del multiculturalismo en algunos sectores, en especial los que reivindican su pertenencia étnica, hay una nueva estilización de los cuerpos a través de lo afro. Así, una parte de la redefinición, desestigmatización y reivindicación de lo Negro $^{28}$ y lo afrocolombiano pasa por la reivindicación de la estética afro que se recrea de manera permanente.

En este sentido, los cuerpos se vuelven un lugar de resistencia a la imposición de la estética occidental hegemónica. Uno de los elementos que traduce bien esta estilización de los cuerpos femeninos afro es el cabello. De hecho, como lo cuentan varias de nuestras entrevistadas, el cabello se vuelve un lugar central de su construcción como mujeres Negras puesto que pasa de ser un elemento molesto, maltratado y traumático a ser un elemento de orgullo y de reivindicación. Con base a lo anterior, el llamado racismo solapado, es decir un racismo no explícito, y sin embargo muy presente (Cadena, 2001; Oboler, 1996), no es más gentil que el racismo abierto sino que puede ser incluso más agresivo porque es muy dificil de atacar. En este sentido, argumentamos que en cierto modo, el multiculturalismo apoya el racismo solapado siendo que aun cuando el racismo está socialmente condenado y se considera políticamente incorrecto, el hecho de denunciar las expresiones racistas muchas veces conduce a que se cuestione a quien las denuncia y no a quienes las cometen.

De este modo y bajo la valoración máxima que se le da a la tolerancia, cuestionar los comportamientos racistas y las estructuras sociales y cognitivas racializadas se vuelve una acción difícil y muy costosa, tanto emocionalmente como socialmente, tal como lo describen mis entrevistadas que decidieron desafiar estas estructuras racistas a través de su estética, trabajo y relaciones sociales. Eso implica tanto enfrentar las situaciones y a sus interlocutores, como explicarles los fundamentos (el sentido común racializado) y las implicaciones (el racismo y la discriminación racial) de sus comportamientos. En otras palabras, el sujeto se convierte entonces en la pedagoga de todos los días.

\footnotetext{
${ }^{28}$ El uso de la N mayúscula indica que se trata de una categoría de auto-identificación.
} 


\section{A modo de conclusión: un proyecto planteado que queda por realizar}

En este artículo argumentamos que el multiculturalismo, si bien reconoce -aunque tímidamente- el problema del racismo en la sociedad colombiana, no está planteado como un problema de la sociedad en su conjunto sino como un problema de sus víctimas -en este caso la población afrocolombiana-y de los individuos que actúan, de manera explícita y se asumen de manera voluntaria, como racistas. Bajo esta perspectiva, no habria una ruptura entre la ideología del mestizaje y el multiculturalismo pues ambos -aunque de modos diferentes-, omiten el problema de las relaciones raciales y sus efectos. Sin embargo, si bien el multiculturalismo en sí no reconoce ni denuncia la persistencia de las estructuras sociales y de poder racializadas, planteamos que sí abre varios espacios que permiten una agencia por parte de los sujetos racializados como negros que a su vez transforman, redefinen y amplian el multiculturalismo y sus alcances.

Al mismo tiempo, planteamos que las personas racializadas como negras que no han sido originalmente incluidas por el multiculturalismo son las que lo desafian y cuestionan las estructuras sociales racializadas. Estas personas que transcienden las fronteras establecidas por la etno-racialización nos muestran hasta qué punto la racialización proviene y concierne a toda la sociedad y por lo tanto, que al ser vista y tratada como un problema de la población negra, no puede ser remediada por completo.

Para acabar con la racialización discriminatoria habria que ir más allá de la penalización del racismo propuesta por la Ley Antidiscriminación pues, tal como lo subraya Glenn Loury en The Anatomy of Racial Inequality (2002), eliminar la discriminación in contract (en relaciones formales, a saber relaciones laborales etc.) no es y no puede ser suficiente. Es necesario eliminar la discriminación in contact (en relaciones sociales informales, de amistad, familiares, etc.) que a su vez, no desaparecerá mientras persista la estigmatización racial. Como lo explica el autor: "Discrimination is about how people are treated, stigma is about who, at the deepest cognitive level, they are understood to be" (Loury, 2002, p. 167).

Sin embargo, la eliminación de la estigmatización implica unas reformas estructurales politicas, sociales e incluso cognitivas profundas que requieren tanto del reconocimiento de la existencia del racismo, como del reconocimiento de la responsabilidad que la sociedad y cada 
uno de sus miembros tiene en su reproducción, lo que implica a su vez repensar los fundamentos mismos de la sociedad colombiana. En este sentido, consideramos que el multiculturalismo corre el riesgo de perpetuar la racialización a través de nuevos mecanismos, pero que al mismo tiempo contiene un potencial para atacarla.

Se trata, desde nuestro punto de vista, de una elección entre la tolerancia y el respeto, un dilema que todavía no ha sido resuelto, tal como lo muestra por ejemplo la política educativa multicultural y su principal instrumento: la Cátedra de Estudios Afrocolombianos. Mientras la Cátedra afrocolombiana siga siendo percibida como un instrumento para la población Negra y afrocolombiana, alimentando así las relaciones de tolerancia que no permiten un verdadero conocimiento de los que son vistos como Otros, más que romper con las estructuras racializadas, esta hará parte de ellas. Sin embargo, al concebir la Cátedra afrocolombiana como un mecanismo de analizar y repensar la sociedad a través y desde la población afrocolombiana, se hace posible replantear la sociedad y sus fundamentos y con esto se abre el camino hacía el respeto, no del Otro, sino de los diferentes miembros de la sociedad colombiana.

\section{Referencias}

Agudelo, C. (2004). Politique et populations noire en Colombie. Paris: L'Harmattan. Aguilar, M. A. (2011). Blanco Porcelana.Trabajo no publicado.

Becerra, L. M., y Ruiz, P. L. C. (2008). Vidas ante la adversidad: Informe sobre la situación de los derechos humanos de las Mujeres Afrocolombianas en la situación de desplazamiento forzado. Bogotá: Afrodes.

Bocarejo, D., y Restrepo, E. (2011). Introducción. Revista Colombiana de Antropología, 47(2), 7-13.

Bonilla-Silva, E. (1997). Rethinking Racism: Toward a Structural Interpretation. American Sociological Review, 62 (3), 465-480.

Cadena, M. (2001). The Racial Politics of Culture and Silent Racism in Peru. Racism and Public Policy Conference. Durban, South Africa: United Nations Reserch Institutefor Social Development. Recuperado de http://www.unrisd. org/80256B3C005BCCF9/ (httpAuxPages)/EE7EB1E30A96C11 1F80256B6D0 0578643/\$file/dcadena.pdf

Cárdenas, J. C., Ñopo, H., y Castañeda, J. L. (2012). Equidad en la diferencia: Politicas para la Movilidad Social de Grupos de Identidad Mision de Movilidad Social y Equidad. Bogota: Universidad de los Andes - Facultad de Economia - CEDE. 
CONPES, Consejo Nacional de Política Económica y Social (2002). CONPES 3169: Política para la población afrocolombiana. Bogotá: Ministerio Nacional de Planeación.

CONPES, Consejo Nacional de Política Económica y Social (2010). CONPES 3660: Politica para promover la igualdad de oportunidades para la población Negra, Afrocolombiana, Palenquera y Raizal. Bogotá: Ministerio Nacional de Planeación.

Crenshaw, K. (1991). Mapping the Margins: Intersectionality, Identity Politics, and Violence against Women of Color. Stanford Law Review, 43 (6), 1241-1299.

Cunin, E. (2004). Métissage et multiculturalisme en Colombie (Carthagène). Paris: L'Harmattan.

DANE, Departamento Administrativo Nacional de Estadística. (2010). Análisis regional de los principales indicadores sociodemográficos de la comunidad afrocolombiana e indigena a partir de la información del censo general de 2005. Bogotá: Departamento Administrativo Nacional de Estadística.

Essed, P. (1991). Understanding Everyday Racism: an Interdisciplinary Theory (Vol. 2). Newbury Park: Sage publications.

Fanon, F. (1973). Piel negra, máscaras blancas. Buenos Aires: Abraxas.

Goldberg, D. T. (1993). Racist Culture: Philosophy and the Politics of Meaning. Oxford: Blackwell.

Gunaratnam, Y. (2003). Researching Race and Ethnicity: Methods, Knowledge and Power. London: Sage Publications Ltd.

Hall, S. (2000). The Multicultural Question. Paper presented at the The Political Economy Research Centre Annual Lecture, Firth Hall Sheffield. En B. Hesse (Ed.) Un/settled Multiculturalisms (pp. 209-245). London: Zed Books.

Hernández, F. G. G. (2010). Vivir en un mundo de "blancos": experiencias, reflexiones y representaciones de 'raza' y clase de personas negras de sectores medios de Bogotá D.C. Bogotá: Universidad Nacional de Colombia.

Hoffmann, O., Barbary, O., y Cunin, E. (2004). Cité, ethnicité: les configurations de l'ethnicité noire en ville. En F. Dureau, O. Barbary, V. Goueset, O. Pissoat y T. Lulle (Eds.). Villes et sociétés en mutation. Lectures croisées sur la Colombie (pp. 184-228). Paris: Antropos.

Hooks, B. (1984). Black Women: Shaping Feminist Theory. En B. Hooks. Feminist Theory: From Margin to Center (pp. 1-15). Boston: South End Press.

Kim, C. J. (2004). Unyielding Positions: A Critique of the 'Race' Debate. Ethnicities, 4 (3), 337-355.

Loury, G. C. (2002). The Anatomy of Racial Inequality. Harvard: Harvard University Press.

Lozano, B. R. L. (2011). El feminismo no puede ser uno porque las mujeres somos diversas. Aportes a un feminismo negro decolonial desde la experiencia 
de las mujeres negras del pacífico colombiano. La Manzana de la Discordia, 5 (2), 7-24.

Lozano, B. R. L., y Sepúlveda, B. D. C. P. (2005, 18-20 de octubre). Memoria y Reparación: ¿Y de ser mujeres negras qué? .Artículo presentado en GEASCES 20 años. Afroreparaciones: Memoria de la Esclavitud y Justicia Social Contemporánea. Diversos puntos de vista. Cartagena de Indias, Colombia.

Lozano, F. A., Hurtado, I. P., y Silva, J. S. (2012). Desplazamiento creciente y crisis humanitaria invisible. Bogotá: CODHES.

Lugones, M. (2008, julio-diciembre). Colonialidad y Género. Tabula Rasa, 9, 73-101.

Oboler, S. (1996). El mundo es racista y ajeno: Orgullo y prejuicio en la sociedad limeña contemporánea. Lima: Instituto de Estudios Peruanos.

Oyěwúmí, O. (1997). The Invention of Women: Making an African Sense of Western Gender Discourses. Mineapolis: University of Minnesota Press.

Prado, F. M. P. (2012). Dosier de proyectos. Texto no publicado.

Quijano, A. (2000, enero-febrero). ¡Qué Tal Raza! Revista Venezolana de Economía y Ciencias Sociales, 6 (1), 37-45.

Rosero-Labbé, C. M., Laó-Montes, A., y Garavito, C. R. (Eds.). (2010). Debate sobre ciudadanía y politicas raciales en las Américas Negras. Bogotá: Universidad del Valle y Universidad Nacional de Colombia.

Salamanca, L. J. G., y Gómez, F. B. (2010). Panorama socioeconómico y politico de la población afrocolombiana, raizal y palenquera: Retos para el diseño de politicas públicas. Bogotá: Ministerio de Cultura.

Salamanca, L. J. G., y Gómez, F. B. (2012). El reto ante la tragedia humanitaria del desplazamiento forzado: Superar la exclusión social de la población desplazada. Bogotá: Comisión de seguimiento a la política pública sobre desplazamiento forzado.

Vigoya, M. V., y Hernández, F. G. (2010). Género y generación en las experiencias de ascenso social de personas negras en Bogotá. Maguaré, 24, 99-130.

Wade, P. (1993). Blackness and Race Mixture: The Dynamics of Racial Identity in Colombia. London: The Johns Hopkins University Press.

Wade, P. (2003). Race and Nation in Latin America: An Anthropological View. En N. P. Appelbaum, A. S. Macpherson y K. A. Rosemblatt (Eds.), Race \& Nation in Modern Latin America (pp. 263-281). Chapel Hill y Londres: The University of North Carolina Press.

Wade, P. (2010). Liberalismo, raza y ciudadanía en Latinoamérica. En C. M. Rosero-Labbé, A. Laó-Montes y C. R. Garavito (Eds.), Debates sobre ciudadanía y politicas raciales en las Américas Negras (pp. 467-486). Bogotá: Programa Editorial. 
Wade, P. (2012, julio-diciembre). Multiculturalismo y racismo. Revista Colombiana de Antropología, 47 (2), 15-35.

Winant, H. (2000). Race and Race Theory. Annual Review of Sociology, 26, 169-185.

WOLA (2010). WOLA's Human Rights Awards Ceremony and Benefit Gala. Recuperado el 10 de julio de 2013 de http://www.wola.org/wola_human_ rights_award. 